\title{
PASADO, PRESENTE Y FUTURO DE LOS HBIM (HERITAGE/HISTORIC BUILDING INFORMATION MODELS)
}

\author{
Pilar Merchán, Santiago Salamanca, María José Merchán, Emiliano Pérez, Ma Dolores Moreno \\ Escuela de Ingenierías Industriales. Universidad de Extremadura. Avda. de Elvas, s/n. 06006 Badajoz, \\ \{pmerchan, ssalamanca, mjmerchan, emilianoph, mdmorenorabel\}@unex.es
}

\begin{abstract}
Resumen
Este trabajo trata de la adaptación experimentada por la metodología BIM (Building Information Modelling) para abordar el modelado y la gestión de edificios históricos, obteniendo lo que se conoce como HBIM (Heritage/Historic Building Information Model). Las siete dimensiones y los niveles de madurez establecidos en BIM, se evalúan y analizan para extrapolarlos al ámbito del patrimonio cultural arquitectónico. Se presenta también una sección sobre la evolución del software BIM y cómo puede utilizarse en el desarrollo de modelos de edificaciones históricas. Finalmente, se hace una revisión de la literatura publicada en los últimos años, mostrando la importancia que el enfoque HBIM ha ganado en los últimos tiempos como una herramienta importante para la gestión integral de los edificios del patrimonio cultural desde un punto de vista multidisciplinar. Esta revisión saca a la luz la falta de soluciones generales para la generación de repositorios de objetos paramétricos para los diferentes estilos arquitectónicos, debido a la amplia gama de posibilidades que se pueden encontrar.
\end{abstract}

Palabras clave: edificios históricos, metodología $\mathrm{BIM}$, adquisición y modelado de datos 3D, software.

\section{INTRODUCCIÓN}

Los modelos BIM engloban los procesos de diseño, construcción y administración de los datos de un edificio a lo largo de su ciclo de vida. Comprenden, tanto la geometría del edificio y sus relaciones espaciales, como las cantidades y propiedades de sus componentes, entre otros elementos, e involucran en el proceso a los agentes del ámbito de la construcción, la arquitectura y la ingeniería.

En las últimas décadas, el sector de la construcción ha incorporado gradualmente estos modelos de información integrada debido a que aportan grandes beneficios y permiten economizar recursos durante el diseño, la planificación y la construcción de nuevos edificios, por lo que empieza a convertirse en una práctica común. Sin embargo, respecto al mantenimiento, la remodelación/restauración o la demolición de edificios existentes, esta metodología apenas se ha utilizado. No obstante, Volk et al. [25] demuestran en una revisión de más de 180 artículos que, aunque la implementación de BIM en edificios existentes todavía es limitada, la investigación sobre ese tema se está intensificando con el fin de aprovechar sus indudables ventajas en la aplicación a este tipo de construcciones. En este campo, sus hallazgos revelan que los principales desafíos son: 1) la automatización de la captura de datos y su implementación para la realización ex novo del modelo BIM correspondiente; 2) actualización y mantenimiento de la información; 3) manejo y modelado de datos no conocidos, objetos y relaciones que se dan en las edificaciones previamente construidas.

Ante un caso especial dentro de esta rama nos encontramos al enfrentarnos con edificios históricos, para los cuales es necesario adaptar los modelos BIM a sus peculiares características. Con ello se obtiene los conocidos como HBIM, modelos de información integrada de edificios patrimoniales, que se constituyen como un nuevo sistema de gestión de la información en el ámbito de las intervenciones en patrimonio cultural.

Hasta ahora, para este tipo de construcciones sólo se dispone de modelos geométricos 3D que muestran sus condiciones físicas. Pero estos datos por sí solos no son suficientes ni muy útiles, ya que no ofrecen la posibilidad de obtener la información de una manera completa, integrada y fácilmente accesible. La necesidad de enriquecer semánticamente los modelos 3D y, con ello, crear un repositorio más completo para cualquier edificio patrimonial se pone de manifiesto por el creciente interés de las investigaciones recientes. Sin embargo, aún nos encontramos en las etapas iniciales puesto que la metodología desarrollada no se ajusta a las peculiaridades constructivas de los edificios históricos [23]. En este trabajo presentamos una revisión de los avances llevados a cabo hasta el momento y señalamos las líneas por las que debe continuar la investigación a partir de ahora. 


\section{LAS DIMENSIONES DEL HBIM}

Con el término "dimensión" nos referimos a la forma particular en la que ciertos tipos de datos están vinculados a un modelo de información. Al agregar sucesivas "dimensiones" se proporciona una comprensión más completa del proyecto de construcción (cómo y cuándo se entregará, cuánto costará, cómo se debe mantener, etc.). Aunque hay algunas discrepancias en la definición y el número de dimensiones en el entorno BIM, parece que la idea más extendida es que existirían siete, que pasamos a resumir a continuación, dando cuenta, además, de la modificación que sufrirían para adaptarse a edificios pertenecientes al patrimonio arquitectónico.

\section{D}

Esta dimensión trata de dibujos y especificaciones. A partir del surgimiento de los modelos BIM, que sustituyeron a los sistemas CAD, los dibujos se generan de forma automática y paramétrica como resultado del diseño 3D. Esto permite mantener la información totalmente actualizada y detectar posibles conflictos en todas las fases del proyecto.

Para generar HBIM, es crucial llevar a cabo una etapa de documentación en la que recopilar toda la información histórica del edificio, lo que a veces puede convertirse en un verdadero desafío. No obstante, la fase de diseño se simplifica, por venir éste impuesto.

\section{D}

En un BIM, la tercera dimensión implica la visualización y coordinación del proyecto, que es la base de esta metodología. Con ello se permite a los diversos colaboradores no solo ver de antemano el edificio en tres dimensiones, sino también actualizar automáticamente estas vistas a lo largo de todo el proyecto. Además, es una herramienta colaborativa muy efectiva pues el modelo 3D contiene datos relevantes para todos los participantes del proceso (inversores, diseñadores, contratistas, gerentes y propietarios). Incluso permite resolver problemas de manera predictiva al mantenerse continuamente actualizada con datos reales y precisos. El más mínimo cambio en esta dimensión se reflejará en las restantes.

La adaptación de esta dimensión a HBIM se centra en la obtención del modelo 3D a partir del uso de las diferentes tecnologías disponibles para capturar la realidad y el procesamiento adecuado de la información obtenida en la dimensión anterior.
4D

La cuarta dimensión aporta datos de planificación a partir de los cuales los diversos participantes puedan extraer y visualizar el progreso de sus actividades a lo largo del proyecto. De este modo se pueden fijar plazos, recomendar operativas de trabajo, calcular tasas de productividad, personal necesario... El uso de la tecnología 4D-BIM puede mejorar la detección de conflictos y favorecer el control de los cambios acaecidos durante la ejecución del proyecto, lo que en algunos casos supondría la reducción de su duración de hasta un $10 \%$.

La planificación de tiempos es aún más interesante cuando se afronta un proyecto de restauración, donde existe una alta probabilidad de encontrar problemas inesperados que obliguen a modificar la idea inicial. La 4D-HBIM permite simular de forma virtual diferentes soluciones y procesos, salvaguardando de este modo la integridad del edificio hasta hallar la mejor medida de actuación posible. Además, almacena la información de la evolución del edificio a lo largo del tiempo. Esta dimensión, bien administrada, puede proporcionar herramientas hasta ahora desconocidas para los equipos multidisciplinares.

\section{D}

En esta dimensión se aborda la estimación de costes, incluyendo listas de cantidades, índices de productividad y gastos en mano de obra. Gracias a su integración dentro del modelo BIM, cualquier cambio de diseño se refleja inmediatamente en el presupuesto. Con ello, se brinda a los equipos pronósticos de flujo de efectivo, análisis precisos y detallados de riesgos y provisión de recursos para los principales subcontratistas.

Para HBIM, además de todo lo anterior, cabe destacar que el hecho de poder contemplar todos los escenarios posibles en el modelo virtual permite evitar discrepancias y minimizar errores. Por otro lado, dado que todos los participantes comparten el flujo de trabajo, los presupuestos se pueden actualizar en tiempo real.

6D

La sexta dimensión aborda la sostenibilidad, esto es, cómo optimizar el consumo de energía. Es un hecho que el sector de la construcción consume el $40 \%$ de la energía mundial y es responsable de producir la mitad del $\mathrm{CO}_{2}$ emitido a la atmósfera. El uso de esta dimensión puede dar como resultado estimaciones de energía más completas y precisas en el proceso de diseño y permitir la medición y verificación durante la construcción, por lo que pueden ser adaptadas a la reglamentación existente contra el cambio climático. 
Esta dimensión necesita una especial dedicación en los entornos HBIM, ya que en la mayoría de los casos los edificios patrimoniales no están sujetos a las normas de certificación de eficiencia energética. Un análisis del consumo de energía en la recreación virtual del edificio proporciona muchas herramientas para encontrar soluciones alternativas con el fin de empezar la adaptación a la normativa.
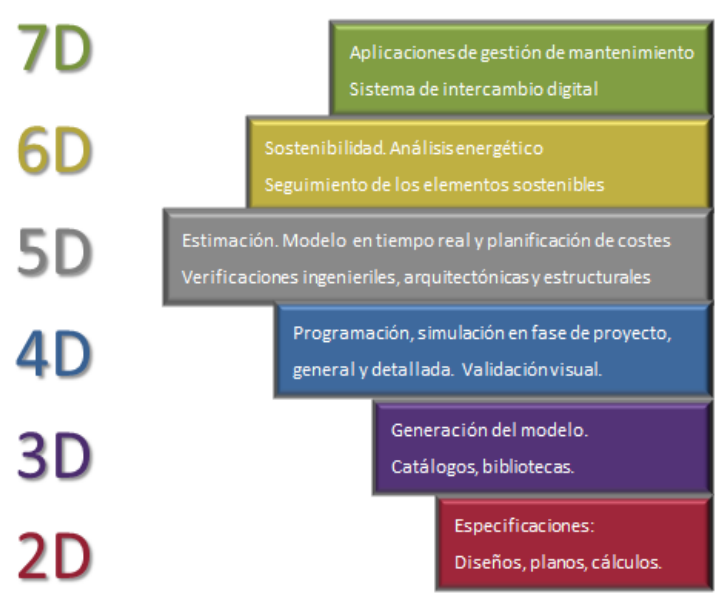

La Figura 1 es un resumen de las siete dimensiones de BIM tal como se han definido hasta ahora [1] frente a nuestra propuesta para HBIM. La longitud de cada barra define la importancia que cada dimensión tiene dentro de BIM / HBIM.

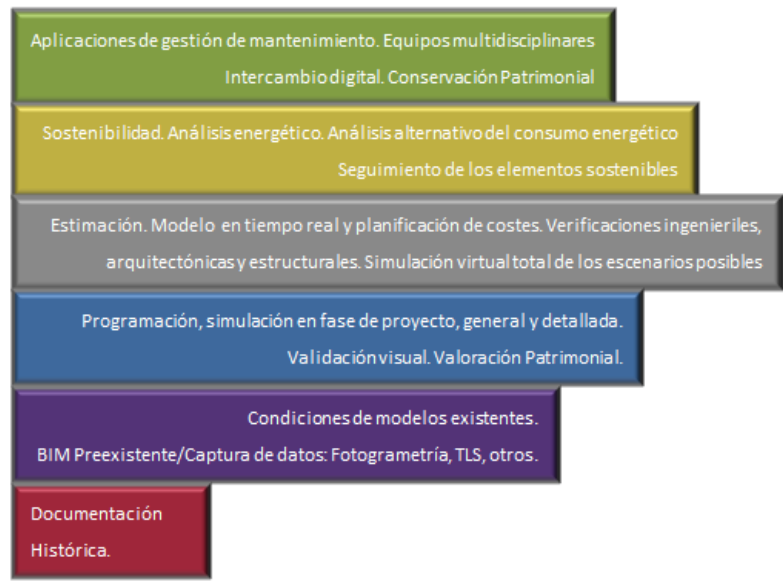

BIM HBIM

Figura 1: Representación de 7D BIM vs. 7D HBIM

$7 \mathrm{D}$

7D-BIM cubre la gestión del edificio a lo largo de su ciclo de vida, por lo que se convierte en el manual a seguir para su uso y mantenimiento desde el momento de la construcción hasta su demolición y reciclaje. Como el período de funcionamiento de un edificio es siempre mucho mayor que el de construcción, los procedimientos de gestión de las instalaciones se convierten en el núcleo de cualquier política BIM, al facilitar el proceso de mantenimiento normativo y preventivo de las instalaciones. Con el uso de esta herramienta, podemos, de modo más fácil y rápido:

- Obtener piezas de repuesto: marcas, productos, dimensiones y composición están especificados.

- Optimizar el ciclo de vida: integrando los datos de esperanza de vida y los costos de reemplazo de equipos, sistemas y componentes.

- Gestionar el mantenimiento.

- Administrar espacios e instalaciones.

Cuando se trata de un edificio patrimonial, el ciclo de vida se reduce al uso, mantenimiento y reparación. Por lo tanto, esta dimensión en HBIM se encarga de la detección y el control de problemas y de la supervisión de la evolución del monumento. Gracias a esta dimensión, los criterios y datos para la correcta preservación del edificio están unificados y a disposición de todos los profesionales responsables de esta tarea.

\section{NIVELES DE MADUREZ HBIM: LA IDEA DE INTEROPERABILIDAD}

Los gobiernos han reconocido que el proceso de transformación de la industria de la construcción en un entorno de trabajo colaborativo BIM será progresivo, con hitos concretos y reconocibles que se definirán en forma de niveles. Éstos se moverán dentro de un rango de 0 a 3 y especifican la madurez alcanzada por los modelos BIM.

\section{Nivel 0}

El nivel 0 es esencialmente el uso del diseño asistido por ordenador (o herramientas CAD) para crear dibujos utilizando software. El intercambio de información ocurre sin estándares ni procesos comunes. Básicamente, se trata de un tablero de dibujo digital que puede considerarse el paso siguiente a generar la información a mano.

\section{Nivel 1}

Es una mezcla de información 2D y 3D, también conocido como CAD administrado. Representa el primer paso hacia el uso de datos 3D para presentar el diseño. En este nivel, los modelos todavía no se comparten entre los miembros del equipo y la colaboración se introduce a través de un entorno de datos común (CDE, Common Data Environment). Es por eso que este nivel se llama coloquialmente 'BIM solitario'. 
Nivel 2

Conocido, generalmente, como nivel de colaboración BIM. Todas las partes crean sus propios modelos en $3 \mathrm{D}$, pero no necesariamente trabajan en un único modelo colaborativo. Para compartir la información de todos ellos se usa un entorno de datos común online. La información de diseño, que puede incluir información de secuencias de construcción (4D) y coste (5D), se sube en un formato de archivo común.

\section{Nivel 3}

Se conforma ya un verdadero modelo de información totalmente integrado, que se aloja y desarrolla en un repositorio centralizado. Todas las partes implicadas pueden acceder y modificar el mismo modelo en tiempo real, por lo que se elimina la capa final de riesgo de información conflictiva. En este nivel de BIM se utilizarán las siete dimensiones.

El Nivel 3 de madurez aún está lejos de alcanzarse, puesto que los sectores de la arquitectura, la ingeniería y la construcción siguen centrados en el soporte, la capacitación y la educación necesarios para alcanzar y refinar sus capacidades en el Nivel 2.

$\mathrm{Si}$ extrapolamos estas consideraciones sobre niveles al HBIM, la madurez alcanzada por el sector todavía se encontraría en el Nivel 1.

El tercer nivel de madurez (colaboración completa entre todas las disciplinas) es el que lleva a la idea de interoperabilidad, entendida ésta como la capacidad de comunicación entre las personas y entre los distintos tipos de software. La interoperabilidad permite analizar los elementos de un edificio de forma multidisciplinar, ofreciendo la posibilidad de unificar la información obtenida a través de diferentes tecnologías, analizadas por diferentes investigaciones y guardadas en diferentes formatos.

Desde el punto de vista de la interoperabilidad, según Murphy et al. [16], HBIM puede considerarse como una nueva biblioteca de objetos paramétricos, basada en datos arquitectónicos históricos y en un sistema de programas multiplataforma con los que mapear dichos objetos en las nubes de puntos. La creación de estas bibliotecas puede ser relativamente fácil cuando el estilo arquitectónico está perfectamente definido y bien documentado, pero puede convertirse en una ardua tarea cuando no lo está, siendo éste un tema apenas abordado hasta ahora. El problema más recurrente que hemos encontrado en la literatura es la ausencia de bibliotecas de objetos paramétricos que faciliten la construcción de HBIM, como sí puede hacerse para edificios de nueva construcción.

\section{CREACIÓN DE MODELOS HBIM}

No es posible hablar ni de BIM ni de HBIM sin tener en cuenta el software que se puede usar para crearlos.
En este sentido, ArchiCAD® fue el primer software BIM para PC, a resultas del trabajo que comenzó Gábor Bojár en 1982 en Graphisoft, la empresa fundada por él que sigue siendo la encargada de comercializarlo hoy día. Sin embargo, el reconocimiento de su importancia para el desarrollo de proyectos BIM no le ha llegado hasta hace pocos años. Uno de los mayores logros de este software es la integración de un entorno de programación en GDL (Geometric Description Language), lenguaje de Graphisoft que permite la creación de objetos paramétricos 2D y 3D.

Años después surge Revit®, que hoy en día es el software más utilizado en diseño BIM. Al igual que ArchiCAD®, Revit® incluye un entorno de programación, en este caso visual, que permite la creación de objetos paramétricos. Sin embargo, la gran contribución de este software fue permitir la introducción del parámetro tiempo en los modelos de los edificios, esto es, implementar la cuarta dimensión. Esto posibilitó simular los procesos de construcción de los edificios.

En el nivel actual de madurez para BIM, el Nivel 2, en el que la interoperabilidad se logra mediante el uso de diferentes programas informáticos que resuelven cada una de las dimensiones de BIM, comunicándose entre sí por medio de formatos compatibles, el formato de intercambio de datos más popular es IFC (Industrial Foundation Classes), que es un estándar abierto y neutral mantenido por buildingSMART®. Desde el lanzamiento de IFC4, se ha aceptado como la norma ISO 16739 [15]. Además, gbXML es ampliamente utilizado, aunque se centra principalmente en la dimensión 6 del BIM [14].

Sin embargo, como se mencionó anteriormente, HBIM aún se encuentra en el nivel 1. En este nivel, se implementan las dimensiones 2D y 3D. Así, mientras que la creación de los modelos BIM se basan en objetos paramétricos simples (paredes, suelos, columnas...) que se integran para obtener el resultado 3D final, para generar un HBIM es necesario comenzar por obtener los datos 3D originales del edificio. Más tarde, estos datos se segmentarán para definir los elementos que finalmente conducirán a la creación de los modelos paramétricos. Por lo tanto, en el caso de HBIM, se realiza un proceso up-down, mientras que en BIM se lleva a cabo un proceso down-up [23].

La forma más común de obtener información 3D de un edificio existente es a través de escáneres láser, que a menudo se complementa con otras metodologías como la fotogrametría. La información proporcionada son nubes de puntos 3D en las que, en la mayoría de los casos, la información de color 
también está integrada. Esta información no se puede usar directamente, ya que los diferentes elementos arquitectónicos para modelar deben identificarse dentro de las nubes de puntos. Actualmente, estos procedimientos se realizan manualmente, antes de importar nubes de puntos tridimensionales en el software comercial, como Revit, puesto que todavía no existe un método de segmentación automático y general que resuelva esta tarea.

Por lo tanto, la segmentación automática de nubes de puntos 3D densas es una de las áreas de investigación más interesantes que se abren actualmente en HBIM. Por otro lado, la creación de modelos paramétricos en HBIM depende de si el estilo arquitectónico en cuestión está documentado o no, como dijimos. Por ejemplo, los estilos clásicos se recogen desde el 1530 A.C. en el libro De Architectura, de Vitrubio, mientras que muchos otros, como los estilos arquitectónicos populares, no están documentados en ningún libro o manual. Esto da lugar al hecho de que las familias paramétricas se pueden realizar fácilmente, e incluso encontrar, en los estilos para los que se posee este tipo de información mientras que, para los estilos no documentados, la creación de modelos es una tarea ardua que consume muchos recursos. Aunque los softwares mencionados tienen entornos de programación que permiten la creación de modelos paramétricos, algunos de ellos bastante potentes, como Dynamo, integrado en Revit®, en el caso de la segmentación de las nubes de puntos aún no se ha automatizado el procedimiento para crear los modelos paramétricos de los elementos que definen los diferentes estilos arquitectónicos, por lo que esta área constituye también una línea de investigación muy interesante.

En resumen, actualmente no existe ningún software que pueda crear HBIM para ningún tipo de estilo arquitectónico de edificios históricos. La solución, si el estilo está documentado, se puede encontrar en el uso de algunos de los programas existentes, con las limitaciones discutidas. En el caso de los estilos no documentados, la creación de HBIM implica el uso de un software específico (por ejemplo, para la adquisición y procesamiento de datos 3D) o algoritmos ad hoc diseñados para la generación del HBIM en cuestión. En la siguiente sección se revisarán algunas de las propuestas hechas al respecto hasta ahora.

\section{REVISIÓN BIBLIOGRÁFICA. PASADO Y PRESENTE DEL HBIM}

Como se dijo antes, la primera etapa en el proceso de generación de un HBIM es la compilación de toda la documentación histórica disponible para el edificio en estudio. A veces esta es la única información factible. Así, vemos como Boeykens S. et al. [6] presentan la generación de HBIM para la sinagoga de Vinohrady en Praga, un edificio muy singular que está completamente destruido, cuyo modelo se hizo exclusivamente a partir de documentación histórica.

De manera similar, Murphy et al. [17] emprenden la construcción de una biblioteca de objetos paramétricos interactivos construidos a partir de los datos arquitectónicos hallados en manuscritos y lo llaman HBIM. El producto final de este HBIM es una colección de modelos 3D completos que incluyen detalles como los métodos de construcción y la composición del material. Con él se pueden crear automáticamente secciones, detalles o proyecciones ortográficas, así como los modelos 3D (con o sin textura) para el análisis y la conservación de objetos, estructuras y entornos históricos.

Pero además de esto, es necesaria una segunda fase de captura de la información geométrica de un edificio existente mediante, como dijimos, el escaneo láser 3D y la fotogrametría /SfM.

No hay dudas sobre el potencial de los escáneres láser, principalmente debido a su capacidad para adquirir gran cantidad de datos espaciales en un período corto. Producen nubes de puntos densas compuestas por miles o millones de vértices, a partir de los cuales es posible generar superficies con un alto grado de detalle y fiabilidad. El continuo desarrollo de aplicaciones informáticas capaces de gestionar con éxito estas nubes de puntos ha mejorado enormemente el rendimiento de esta herramienta en la obtención de resultados dentro del campo de la arquitectura y la arqueología. Sus principales desventajas son la necesidad de contar con un equipo humano experimentado, así como su coste económico.

Por su lado, la fotogrametría es un método basado en imágenes que proporciona información semántica valiosa que mejora los sistemas directos tradicionales en términos de precisión y reducción de tiempo. Sus principales ventajas en comparación con el láser escáner son su capacidad para discriminar información, su facilidad de uso y su bajo costo. Una de sus principales desventajas es el post-proceso requerido, especialmente cuando la textura de los objetos es pobre o sus formas muy complicadas.

Atendiendo a esta tercera dimensión de HBIM, Dore y Murphy [9] generan una nueva biblioteca de objetos paramétricos dinámicos reutilizables, creados en el lenguaje de descripción geométrica (GDL) y diseñados para modelar elementos arquitectónicos clásicos. Para ello, usaron manuscritos históricos y libros de patrones arquitectónicos (2D) junto con la información proporcionada por escáneres láser y datos de imágenes (3D). También proponen en este 
trabajo una fachada de construcción paramétrica completa que se puede utilizar como plantilla para la generación rápida y eficiente de la geometría de la fachada BIM.

En [5], se toma como el caso de estudio la iglesia de il SS.Nome di Maria (Italia), construida en 1748, y gravemente dañada durante un terremoto ocurrido en 2012. En este artículo se propone un enfoque innovador para construir HBIM en 5 pasos a partir de los que se pretende obtener un conjunto de documentos "comprensibles" (dibujos y paneles) para la edificación con los que es posible dar instrucciones sobre procedimientos, funcionamiento y seguridad. La intervención propuesta en cada caso condicionará el modelo digital (nivel de detalle y gestión). Para obtener los datos geométricos precisos, fue necesario utilizar el escáner láser y la fotogrametría, tanto dentro como fuera de la iglesia.

En el mismo espíritu de investigación para la restitución de edificios históricos, en este caso para medir el impacto de la guerra sobre ellos, Dore et al. [10] generan el HBIM de los Cuatro Tribunales en Dublín, que forma la base para el análisis estructural y de conservación de esta construcción cuya vista aún impacta.

En este punto, y a pesar de la corta vida en el uso de la metodología BIM en entornos históricos, se puede esbozar un procedimiento general para generar un HBIM. El proceso comienza con la recopilación de toda la información disponible para el edificio (2DHBIM), lo que incluye la obtención de las nubes de puntos 3D, cuando sea posible, pues nos permitirán la creación de modelos tridimensionales realistas (3D-HBIM). Estas nubes también deben procesarse para segmentar los diferentes elementos que van a ser parametrizados con el fin de generar bibliotecas de objetos paramétricos que conduzcan al modelo HBIM del edificio. Una vez que se ha obtenido el modelo 3D y se ha compilado toda la información no geométrica del edificio, las restantes dimensiones se pueden implementar de acuerdo con los objetivos perseguidos y así tener el modelo final de HBIM.

Pero, como ya se ha señalado, el problema más generalizado que encontramos en la literatura es la ausencia de bibliotecas de objetos paramétricos que faciliten la construcción de HBIM, como sí se tienen para los edificios nuevos. Sin duda es un problema complejo, pues no solo no tenemos una base de referencia, sino que, dada a la variedad de estilos, elementos, ornamentos y otros objetos, la amplitud de estas bibliotecas debe ser formidable. Repasamos en las siguientes líneas los esfuerzos realizados hasta ahora por diferentes autores para generar bibliotecas de objetos paramétricos bien de estilos específicos, bien de edificios particulares.
Aunque Murphy et al. habían introducido el concepto de bibliotecas de objetos paramétricos dentro del proceso de generación de HBIM en 2009 [16], no es hasta 2013 [17] cuando aplican su propuesta para producir la biblioteca paramétrica de elementos arquitectónicos clásicos que se basan en manuales de arquitectura clásica, desde Vitrubio hasta los libros de patrones arquitectónicos del siglo XVIII.

Previamente, Chevrier et al. habían presentado en [8] un estudio sobre las diversas formas geométricas de un elemento arquitectónico dado, representativo de un período y un lugar específicos. Para ello, proponen automatizar la generación de modelos 3D de varios escenarios relacionados y ejemplifican su metodología en los trabajos realizados en edificios residenciales de los siglos XIX y XX de Francia y Canadá. Sin embargo, en el documento no se hace referencia a la compatibilidad de los objetos generados con el software BIM existente.

Apollonio et al. [2] generan una biblioteca de objetos paramétricos de elementos arquitectónicos de estilos clásicos, utilizando como referencia la obra de Andrea Palladio [20]. Los elementos generados, insertados como una familia de Revit, se utilizan para modelar el Palazzo Barbaran en Porto.

Por su parte, Garagnani [12] desarrolla un plug-in específico para Revit, llamado GreenSpider, que examina las nubes de puntos para procesar objetos paramétricos que representan los datos capturados, conectando los puntos en el espacio como si fueran nodos de una tela de araña ideal.

Baik y Boehm [3], por otro lado, generan una biblioteca de objetos paramétricos Hijazi, la HAOL (Biblioteca de Objetos Arquitectónicos Hijazi, en español), como Roshan y Mashrabiyan, elementos muy importantes dentro de la cultura islámica que tienen una fuerte representación en ciudades como Jeddah en Arabia Saudita, Estambul o El Cairo. Los elementos únicos, hasta ahora no clasificados digitalmente, están representados en esta biblioteca.

Existen, además, muchos otros estudios realizados en el contexto de la metodología BIM aplicada a edificios históricos $[4,5,7,11,13,18,19,21,24]$, pero todos son trabajos sobre un edificio específico y para un estilo arquitectónico particular. Ninguno de ellos ofrece una solución general, lo cual es lógico dado el amplio rango de posibilidades que se pueden encontrar cuando se trata de edificios históricos.

\section{CONCLUSIONES}

Pretendemos presentar con este trabajo una visión general del uso que se ha hecho hasta ahora de la 
metodología BIM para la documentación y gestión del patrimonio arquitectónico. Se han definido siete dimensiones para el modelado basado en HBIM a partir de las dimensiones propuestas para BIM y se ha propuesto una representación gráfica que tenga en cuenta la relevancia relativa de cada dimensión dentro del modelo total.

Los niveles de madurez definidos para BIM se han discutido para HBIM, demostrando que también definen la interoperabilidad en este campo. Se ha podido comprobar la madurez de HBIM está en sus estadíos iniciales mientras que BIM está ya en el nivel 2.

Con respecto al software para trabajar en entornos HBIM, se ha evidenciado la falta de idoneidad del software de modelado BIM al tratar con datos 3D complejos de monumentos históricos, así como la necesidad de una plataforma en la que llevar a cabo todo el proceso de flujo de trabajo que involucra la generación de estos modelos en sus 7 dimensiones.

También ha quedado claro que para HBIM, a diferencia de BIM, y debido a la amplia gama de posibilidades que se pueden encontrar, no hay soluciones generales para la generación de bibliotecas de objetos paramétricos.

En términos de investigación, dos líneas importantes perfectamente definidas y con un amplio camino están abiertas a los investigadores: la segmentación automática de las nubes de puntos densas que proporcionan los escáneres láser y la generación de bibliotecas de objetos paramétricos para estilos arquitectónicos no documentados que hasta ahora no se han abordado.

\section{Agradecimientos}

Este trabajo ha sido financiado por el proyecto IB16162 de la Junta de Extremadura y el Fondo Europeo de Desarrollo Regional "Una manera de hacer Europa".

\section{English summary}

\section{PAST, PRESENT AND FUTURE OF HBIM}

\section{Abstract}

This paper deals with the adaptation experienced by the BIM (Building Information Modelling) methodology to approach the modelling and management of historic buildings, obtaining what is known as HBIM (Heritage/Historic Building Information Model). The seven dimensions and maturity levels established in BIM are evaluated and analyzed to be extrapolated to the field of architectural cultural heritage. A section on the evolution of BIM software and how it can be used in the development of historical building models is also presented. Finally, we make a review of the literature published in recent years, showing the importance that the HBIM approach has gained in recent times as an important tool for the integral management of cultural heritage buildings from a multidisciplinary point of view. This review brings to light the lack of general solutions for the generation of repositories of parametric objects for the different architectural styles, due to the wide range of possibilities that can be found.

Keywords: Heritage Buildings, BIM methodology, $3 \mathrm{D}$ data acquisition and modeling, software.

\section{Referencias}

[1] Aouad, G., Lee, A. and Wu S., 2007, Constructing the Future: nD Modeling. Taylor and Francis, London.

[2] Apollonio, F. Gaiani, M., Zheng, S., 2012. BIM-based modeling and data enrichment of classical architectural buildings. SCIRES-IT, 2 (2), pp. 41-62.

[3] Baik, A., Boehm, J., 2017. Hijazi architectural object library (HAOL). The International Archives of the Photogrammetry, Remote Sensing and Spatial Information Sciences, Volume XLII-2/W3, pp. 55-62.

[4] Bassier M., Hadjidemetriou G., Vergauwen M., Van Roy N., Verstrynge E., 2016. Implementation of Scan-to-BIM and FEM for the Documentation and Analysis of Heritage Timber Roof Structures. EuroMed 2016: Digital Heritage. Progress in Cultural Heritage: Documentation, Preservation, and Protection, pp 79-90.

[5] Biagini, C., Capone, P., Donato, V., Facchini, N., 2016. Towards the BIM implementation for historical building restoration sites. Automation in Construction, 71, pp. 74-86.

[6] Boeykens, S., Himpe, C. and Martens, B., 2012. A Case Study of Using BIM in Historical Reconstruction: The Vinohrady synagogue in Prague. Virtual Architecture (1-30), pp. 729-738.

[7] Castagnettia, C., Dubbinib, M., Riccic, P.C., Rivolaa, R., Gianninia, M., Capra, A., 2017, Critical issues and key points from the survey to the creation of the Historical Building Information Model: the 
case of Santo Stefano Basilica, The International Archives of the Photogrammetry, Remote Sensing and Spatial Information Sciences, Volume XLII5/W1, 467-474.

[8] Chevrier, C., Charbonneau, N., Grussenmeyer, P., Perrin, J., 2010. Parametric documenting of built heritage: 3D virtual reconstruction of architectural details. International Journal of Architectural Computing, 2(8), pp. 135150.

[9] Dore, C., Murphy. M., 2014. Semiautomatic generation of as-built BIM façade geometry from laser and image data. Journal of Information Technology in Construction, 19, pp. 2046.

[10] Dore, C., Murphy, M., McCarthy, S., Brechin, F., Casidy, C., and Dirix, E., 2015. Structural simulations and conservation analysis historic building information model (HBIM), The International Archives of the Photogrammetry, Remote Sensing and Spatial Information Sciences, XL-5/W4, pp. 351-357.

[11] Elizabeth, O., Prizeman, C., 2015. HBIM and matching techniques: considerations for late nineteenth- and early twentieth-century buildings. Journal of Architectural Conservation, 21(3), pp.145159.

[12] Garagnani, S., 2013. Building Information Modeling and real world knowledge: A methodological approach to accurate semantic documentation for the built environment. Digital Heritage International Congress (DigitalHeritage), 1, pp. 489-496, IEEE.

[13] Garagnani, S., Gaucci, A., Gruška, B., 2016. From the archaeological record to archaeobim: the case study of the Etruscan temple of uni in marzabotto. Virtual Archaeology Review, 7(15), pp. 77-86.

[14] gbXML Version 6.01 - November 2015. Accessible online at http://www.gbxml.org/Schema_Current_GreenBuildi ngXML_gbXML.

[15] Industry Foundation Classes. Version 4 Addendum 2. Accessible online at http://www.buildingsmarttech.org/ifc/IFC4/Add2/html/.

[16] Murphy, M., McGovern, E. and Pavia, S, 2009. Historic building information modelling (HBIM). Structural Survey, 27(4), pp. 311-327.
[17] Murphy, M., McGovern, E. and Pavia, S., 2013. Historic Building Information Modelling Adding intelligence to laser and image based surveys of European classical architecture. Journal of Photogrammetry and Remote Sensing, 76, pp. 89102.

[18] Nieto, J.E. Moyano, J.J., 2014. El Estudio Paramental en el Modelo de Información del Edificio Histórico o "Proyecto HBIM." Virtual Archaeology Review, 5 (11), pp. 73-85.

[19] Nieto, J. E., Moyano, J. J., Rico, F., \& Antón, D., 2016. Management of built heritage via the HBIM Project: A case study of flooring and wall tiling. Virtual Archaeology Review, 7 (14), 1-12.

[20] Palladio A., 1570. I Quattro Libri dell'Architettura. Venezia. The Four Books on Architecture translation by Isaac Ware 1738, Dover Publications, New York.

[21] Quirk, V., 2012. A Brief History of BIM. Accessible online at http://www.archdaily.com/302490/a-brief-history-ofbim.

[22] Rowland, I. D., \& Howe, T. N. (Eds.). (2001). Vitruvius:'Ten Books on Architecture'. Cambridge University Press.

[23] Saygi, G. and Remondino, F., 2013. Management of Architectural Heritage Information in BIM and GIS: State-of-the-art and Future Perspectives. International Journal of Heritage in the Digital Era, 2(4), pp. $695-713$.

[24] Yang X., Koehl M., Grussenmeyer P., 2017. Parametric modelling of as-built beam framed structure in BIM environment. International Archives of the Photogrammetry, Remote Sensing and Spatial Information Sciences, Volume XLII-2-W3-651.

[25] Volk, R., Stengel, J., and Schultmann, F., 2014. Building Information Modeling (BIM) for existing buildings-Literature review and future needs. Automation in Construction, 38, pp.109-127.

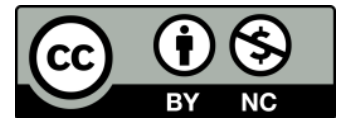

(C) 2018 by the authors. Submitted for possible open access publication under the terms and conditions of the Creative Commons Attribution CC-BY-NC 3.0 license (https://creativecommons.org/licenses/by-nc/3.0). 